\title{
Comparative Analyses of Scylla Olivacea Gut Microbiota Composition and Function Suggest the Capacity for Polyunsaturated Fatty Acid Biosynthesis
}

\section{Nyok-Sean Lau ( $\square$ nyoksean@usm.my )}

Universiti Sains Malaysia

\section{Seng Yeat Ting}

Universiti Sains Malaysia

\section{Ka-Kei Sam}

Universiti Sains Malaysia Janaranjani M.

Universiti Sains Malaysia

\section{Swe Cheng Wong}

Universiti Sains Malaysia

\section{Xugan Wu}

Universiti Sains Malaysia

Khor Waiho

Universiti Sains Malaysia

\section{Hanafiah Fazhan}

Universiti Sains Malaysia

\section{Alexander Chong Shu-Chien}

Universiti Sains Malaysia https://orcid.org/0000-0003-3014-442X

\section{Research Article}

Keywords: Gut microbiota, metagenomics, mud crab, Scylla olivacea, polyunsaturated fatty acid

Posted Date: January 17th, 2022

DOI: https://doi.org/10.21203/rs.3.rs-1249516/v1

License: (c) (i) This work is licensed under a Creative Commons Attribution 4.0 International License. Read Full License 


\section{Abstract}

Multiple studies have linked lipid metabolism with intestinal bacterial structure in aquatic organisms. However, the possibility of the gut microbiota participating in synthesizing beneficial long-chain polyunsaturated fatty acid (LC-PUFA) remains vague. We profiled the gut microbiota of mud crab Scylla olivacea fed with either a LC-PUFA rich diet (FO), a LC-PUFA-poor but C18-PUFA substrate rich diet (LOCO), and diet with a similar profile as LOCO but with the inclusion of an antibiotic, oxolinic acid. Additionally, the microbiota of wild-caught $S$. olivacea was also analyzed. Compared to diet FO treatment, crabs fed diet LOCO contain a higher proportion of Proteobacteria, represented by Vibrio and Shewanella, two known taxonomy groups with PUFA biosynthesis capacity. Annotation of metagenomic datasets also suggests functional contribution by these two genera in terms of the KEGG pathway of unsaturated fatty acids biosynthesis and the keto-acyl synthase domains in the microbiota of LOCO treatment. Diet LOCOAB impeded the presence of Vibrio and Shewanella within the gut, and concurrently, the abundance of PUFA biosynthesis pathway-related genes. However, there was an increase in the function of shortchain fatty acids production within this diet, which was accompanied by a shift towards the abundance of phyla Bacteroidota and Spirochaetota, represented by Sunxiuqinia and Carboxylicivirga and Sediminispirochaeta, respectively. Collectively, these results exemplified bacterial communities and their corresponding PUFA biosynthesis pathways in the microbiota of a farmed crustacean species.

\section{Introduction}

Long-chain polyunsaturated fatty acids (LC-PUFA), including eicosapentaenoic acid (EPA; 20:5n-3), docosahexaenoic acid (DHA; 22:6n-3), and arachidonic acid (ARA; 20:4n-6), are essential for proper cellular, neuronal, cardiovascular, and anti-inflammatory activities [1]. While humans can biosynthesize LC-PUFA from the C18 polyunsaturated fatty acids (PUFA), the consumption of n-3 LC-PUFA, mainly from aquatic food products is necessary [2,3]. The biosynthesis of LC-PUFA requires the fatty acyl desaturases (Fads) and elongase of very long-chain fatty acid (Elovl) enzymes to desaturate and elongate the fatty acyl chain, respectively [4]. The capacity for LC-PUFA biosynthesis varies among primary producers and consumers and is dependent on ecosystem-based differences and the Fads/Elovl genetic machinery [5]. Due to the disparity in the availability of LC-PUFA between different types of ecosystems, species living in terrestrial or freshwater habitats could acquire enhanced LC-PUFA biosynthesis machinery or modifications of foraging behaviours [5]. In natural habitats, an incomplete LC-PUFA biosynthesis pathway could possibly be compensated by selective feeding of prey with higher LC-PUFA content or genetic modification of LC-PUFA biosynthesis enzymes to enhance biosynthesis [6, 7].

Among invertebrates, studies have highlighted the capacity for LC-PUFA production in mollusks and echinoderms with the discovery and validation of the Fads/Elovl orthologs $[8,9]$. The extent of de novo LC-PUFA biosynthesis in crustaceans is still unclear due to the vast diversity of this phyla. A complete repertoire of Fads and Elovl for LC-PUFA biosynthesis was only recently shown in a harpacticoid copepod, T. californicus [10]. In decapods, while the presence of functional Elovl has been reported from mud crab Scylla olivacea [11, 12] and swimming crab Portunus trituberculatus [13], the actual existence of 
functional Fads remains contentious [14]. The vastly diversified Crustacea subphylum includes the Scylla mudcrabs, which have ecological and economical importance in mangroves of the Indo-Pacific region [15]. As aquaculture candidate species, Scylla crabs possess fast growth rate, large size, high reproductive capacity, and adaptability to different farming systems. In many countries however, Scylla farming still depends on freshly prepared wet feed [16]. Knowledge on the capacity for LC-PUFA biosynthesis in Scylla will facilitate the formulation of effective formulated feeds.

The gut microbiome is integral for the overall gut function, immunity, and nutrient processing of the host [17]. The relationships between gut microbiota and various aspects of lipid metabolism such as deposition, digestibility, accumulation, adsorption, synthesis, and obesity have been investigated $[18,19]$. Studies in invertebrates showed the influence of different dietary fatty acids on gut microbiota profile [20, 21]. There is a complex interplay between host dietary lipid intake, gut microbiota characteristics, and tissue fatty acid composition [22]. It is increasingly recognized that PUFA-synthesizing bacteria genera are present in the microbiota of aquatic animals $[23,24]$. While decapods rely on endogenous biosynthesis activities and/or exogenous dietary intake for the supply of LC-PUFA, it is not inconceivable that the host gut microbiota may play a requisite role.

In view of our interest to elucidate a potential role of gut microbiota in LC-PUFA biosynthesis, we fed experimental diets with limited or high levels of LC-PUFA, coupled with the inclusion of antibiotic to juvenile mud crab $S$. olivacea for eight weeks. At end of the feeding trial, animals were dissected for metagenome profiling of the gut microbiota and analysis of fatty acid composition of crab tissues. Overall, the changes in the dynamics of the gut microbiota unveiled the involvement of bacterial groups with LC-PUFA biosynthesis function in S. olivacea.

\section{Material And Methods}

\section{Experimental Animals and Diets}

Fresh male crabs (weight $124 \pm 14.8 \mathrm{~g}$; carapace length $9 \pm 0.3 \mathrm{~cm}$ ) were purchased from local fishermen. Upon arrival, three crabs were dissected immediately to remove the digestive tract and designated as wild crabs (WT). The remaining crabs were acclimatized for two weeks and fed a standard commercial diet. Twenty-seven crabs in the inter-molt stage were randomly distributed in a density of one individual per glass aquarium $(0.60 \mathrm{~m} \times 0.31 \mathrm{~m} \times 0.30 \mathrm{~m})$. These tanks were maintained in a recirculating system with water parameters of $18-24$ ppt salinity, $25-29^{\circ} \mathrm{C}, \mathrm{pH} 7.5-8.3$, and dissolved oxygen $>5 \mathrm{mg} / \mathrm{L}$ and $80 \%$ water change every $48 \mathrm{~h}$.

Three isonitrogenous and isolipidic diets were formulated (Table S1). Diet FO utilized fish oil, whereas LOCO used a 1:1 blend of linseed oil and corn oil as primary lipid sources. Diet LOCOAB has a similar formulation as LOCO, with the inclusion of $75 \mathrm{mg} / \mathrm{kg}$ oxolinic acid. This concentration was based on a preliminary PCR amplification of the 16S rRNA gene to quantify bacterial load [25] (Fig. S1). The fatty acid composition of the three diets reflects their dietary lipid source, with diet FO enriched in LC-PUFA and 
diets LOCO and LOCOAB enriched in C18 PUFA but devoid of LC-PUFA (Table S2). Nine replicate tanks were allocated to each experimental diet. The crabs were fed twice daily till satiation for eight weeks. At the end of the trial, three crabs were randomly collected from each treatment group for metagenomic and fatty acid composition analyses. Method for fatty acid extraction and composition analysis are detailed in Supplementary Note 1.

\section{Sample Collection And Dna Extraction}

Animals were euthanized at $-20^{\circ} \mathrm{C}$ for $20 \mathrm{~min}$, washed, and disinfected with $75 \%$ ethanol. Digestive tracts were aseptically removed, followed by the collection of gut contents and intestinal mucosa into sterile tubes. Genomic DNA was extracted using the DNeasy PowerSoil kit (Qiagen, Germany). The quality and quantity of isolated DNA were assessed using agarose gel electrophoresis and NanoDrop spectrophotometer (Thermofisher Scientific, USA).

\section{6s Rrna Amplicon Sequencing And Analyses}

The V3-V4 variable region of the 16S rRNA gene was amplified using the primers 341F (5'CCTACGGGNGGCWGCAG-3') and 806R (5'-GCACTACHVGGGTWTCTAAT-3'). Libraries for 16S rRNA sequencing were prepared as described previously [26]. Sequencing was performed on an Illumina MiSeq platform generating 250-bp paired-end reads. The 16S rRNA sequencing data were analyzed using the QIIME pipeline (v1.9.1) [27]. Raw sequencing data were pre-processed using Trimmomatic (v0.36) to remove adapter sequences and low-quality bases. Paired-end reads were merged using FLASH, and chimeric sequences were removed using the UCHIME algorithm [28]. Sequences were clustered into operational taxonomic units (OTUs) using UPARSE (v7.1) [29] with a 97\% similarity cut-off. The representative sequences from each OTU were taxonomically classified against the SILVA database [30] using Ribosomal Database Project classifier (v2.2) [31] with a confidence threshold of 70\%. Alpha diversity was estimated using richness (Chao1 index) and diversity (Simpson and Shannon index) parameters. Non-metric multidimensional scaling (NMDS) of the Bray-Curtis distance and principal coordinate analysis (PCoA) of the weighted UniFrac distance was applied to analyze the beta diversity. An unweighted pair group method with arithmetic mean tree was constructed using QIIME. Venn diagram, species accumulation, and rank abundance curves were generated using the R project for statistical computing. A linear discriminant analysis (LDA) effect size (LEfSe) [32] was performed to present the enrichment of bacterial taxa between groups.

\section{Shotgun Metagenomic Sequencing And Analyses}

Sequencing libraries were prepared using the TruSeq Nano DNA library preparation kit (IIlumina, USA) and sequencing was performed on an Illumina NovaSeq 6000 platform at 150-bp paired-end. The raw sequencing data were processed to filter low-quality reads and adapters using Trimmomatic. Taxonomic 
classification by $k$-mer based lowest common ancestor approach was performed using Kraken2 (v2.0.6) [33]. De novo assembly of the clean reads was carried out using Megahit (v1.1.1) [34] with minimum contig length set to $500 \mathrm{bp}$. The genome of a closely related species, Portunus trituberculatus [35], was used as reference sequence to remove host contigs from the metagenome assemblies. Open reading frames (ORFs) were predicted using MetaProdigal (v2.6.3) [36], and the ORFs were clustered using CDHIT (v4.8.1) [37] at $95 \%$ identity and $90 \%$ coverage. Annotation of the unique gene set was performed through BLASTp against the NCBI non-redundant protein (NR), Clusters of Orthologous Groups, and Kyoto Encyclopedia of Genes and Genomes (KEGG) databases at an e-value threshold of 1e-5. Antibiotic resistance genes in the metagenomes were predicted by a search against the Comprehensive Antibiotic Resistance Database [38]. The hits to keto-acyl synthase sequences were retrieved from BLASTp of the unique gene set to the NCBI NR database. Maximum likelihood analysis of KS was carried out using RAxML (v8.2.12) [39] with 1,000 bootstraps and GAMMA+VT model.

\section{Results}

\section{Crab Survival and Growth}

There was no mortality in dietary treatments of FO and LOCO throughout the feeding trial, while $77 \%$ survival occurred in diet LOCOAB treatment (Table S3). Diet LOCOAB also resulted in lower percentage of molting and specific growth rate as compared to diets FO and LOCO. This also resulted in higher feed conversion ratio value in $\mathrm{LOCOAB}$ treatment, despite similar feed intake between crabs from different dietary treatments.

\section{Overview Of The Metagenomic Sequencing Data}

A total of 559 million paired-end reads, with an average of 47 million reads per sample was generated from shotgun metagenomic sequencing of the WT, FO, LOCO, and LOCOAB samples (Table 1). After trimming, 537 million clean reads were assembled into 52,275-85,353 contigs with a total length of 94$174 \mathrm{Mb}$ and $\mathrm{N} 50$ contig length of 2,615-5,843 bp. A non-redundant protein-coding gene set containing $\sim 1.89$ million ORFs was predicted from the 12 metagenomes. 
Table 1

Summary of the shotgun metagenomic sequencing data of $S$. olivacea gut microbiota from wild-caught crabs (WT), crabs fed diet FO, LOCO, or LOCOAB.

\begin{tabular}{|llllllll|}
\hline Samples & $\begin{array}{l}\text { No. of raw } \\
\text { reads }\end{array}$ & $\begin{array}{l}\text { No. of } \\
\text { clean } \\
\text { reads }\end{array}$ & $\begin{array}{l}\text { No. of } \\
\text { contigs }\end{array}$ & $\begin{array}{l}\text { N50 } \\
\text { (bp) }\end{array}$ & $\begin{array}{l}\text { Assembly } \\
\text { size (Mb) }\end{array}$ & $\begin{array}{l}\text { No. of } \\
\text { ORFs }\end{array}$ & $\begin{array}{l}\text { Average ORF } \\
\text { length (bp) }\end{array}$ \\
\hline WT1 & $43,931,876$ & $42,488,724$ & 53,513 & 2,615 & 95.30 & 126,021 & 665 \\
\hline WT2 & $48,522,778$ & $46,858,652$ & 56,482 & 3,018 & 105.76 & 137,920 & 673 \\
\hline WT3 & $49,736,880$ & $48,278,740$ & 52,275 & 2,815 & 93.95 & 124,356 & 666 \\
\hline FO1 & $54,927,728$ & $52,825,174$ & 85,353 & 5,395 & 173.66 & 218,756 & 699 \\
\hline FO2 & $44,267,802$ & $42,378,718$ & 71,426 & 5,843 & 156.65 & 193,531 & 716 \\
\hline FO3 & $40,904,536$ & $39,423,340$ & 64,900 & 5,791 & 147.33 & 180,376 & 723 \\
\hline LOCO1 & $48,501,854$ & $46,462,276$ & 66,117 & 4,796 & 133.61 & 169,367 & 702 \\
\hline LOCO2 & $45,912,916$ & $44,165,248$ & 61,136 & 4,742 & 126.62 & 159,463 & 709 \\
\hline LOCO3 & $47,387,810$ & $45,414,300$ & 65,324 & 4,939 & 132.59 & 167,666 & 705 \\
\hline LOCOAB1 & $49,051,030$ & $46,775,560$ & 63,356 & 2,894 & 110.96 & 142,647 & 676 \\
\hline LOCOAB2 & $42,311,286$ & $40,324,828$ & 60,475 & 2,642 & 102.10 & 133,164 & 669 \\
\hline LOCOAB3 & $43,761,230$ & $41,718,530$ & 58,772 & 2,824 & 101.76 & 131,813 & 672 \\
\hline
\end{tabular}

The 16S rRNA amplicon sequencing produced 2.95 million reads from the 12 individual samples, ranging from 212,812 to 292,709 reads for each sample (Table 2). These sequences were delineated into 18,514 OTUs, corresponding to an average of 1,543 OTUs per sample. For community richness and diversity comparison, alpha diversity parameters were calculated from the proportion of OTUs. The Chao 1 index, an indicator of microbiota community richness, varied from 1,410 to 2,124. Among the different treatments, FO and LOCO samples exhibited higher OTUs and Chao1 index values than WT and LOCOAB. Similarly, Shannon index value was also highest in FO. The Good's coverage estimator of sequencing completeness ranged from 0.998 to 0.999 , indicating high species coverage within the samples. Additionally, the species accumulation curve appeared to flatten after 8 , indicating that the number of samples was sufficient to reflect the species abundance (Fig. S2A). LOCO samples showed the widest and smoothest curve in rank abundance analysis, indicating the highest species richness and, concomitantly, the species uniformity among sample groups (Fig. S2B). Species richness and community evenness were lowest in the LOCOAB samples. 
Table 2

Summary of the $16 \mathrm{~S}$ rRNA sequencing data and alpha-diversity indexes of $S$. olivacea gut microbiota from wild-caught crabs (WT), crabs fed diet FO, LOCO, or LOCOAB.

\begin{tabular}{|lllllll|}
\hline Samples & No. of reads & OTU & Shannon & Simpson & Chao1 & Good's coverage \\
\hline WT1 & 292,709 & 1,489 & 2.85 & 0.24 & 1,820 & 0.999 \\
\hline WT2 & 268,093 & 1,482 & 3.03 & 0.19 & 1,822 & 0.999 \\
\hline WT3 & 220,682 & 1,332 & 2.75 & 0.25 & 1,709 & 0.998 \\
\hline FO1 & 212,812 & 1,648 & 3.92 & 0.05 & 1,977 & 0.998 \\
\hline FO2 & 244,073 & 1,746 & 3.90 & 0.06 & 2,019 & 0.998 \\
\hline FO3 & 262,948 & 1,815 & 4.04 & 0.05 & 2,107 & 0.999 \\
\hline LOCO1 & 214,435 & 1,752 & 3.80 & 0.08 & 2,053 & 0.998 \\
\hline LOCO2 & 241,125 & 1,800 & 3.45 & 0.14 & 2,124 & 0.998 \\
\hline LOCO3 & 247,755 & 1,874 & 3.75 & 0.10 & 2,123 & 0.999 \\
\hline LOCOAB1 & 264,262 & 1,214 & 3.38 & 0.10 & 1,440 & 0.999 \\
\hline LOCOAB2 & 217,363 & 1,144 & 3.29 & 0.11 & 1,410 & 0.999 \\
\hline LOCOAB3 & 261,232 & 1,218 & 3.42 & 0.09 & 1,462 & 0.999 \\
\hline
\end{tabular}

The PCOA and NMDS analyses of beta-diversity were performed to visualize the differences in bacterial communities among samples (Fig. 1A and 1B). Principal component 1 (PC1) and PC2 accounted for $72.34 \%$ and $17.38 \%$ of the composition variance, respectively, reflecting the dissimilarity in the bacterial community composition among different dietary groups. Additionally, the hierarchical clustering tree showed a clear distinction among these groups while the replicates within each group are consistently grouped (Fig. 1C). The Venn diagram showed 684 shared OTUs among the four groups and 389, 133, 165, and 153 unique OTUs within the WT, FO, LOCO, and LOCOAB groups, respectively (Fig. 1D). Treatments FO and LOCO shared the highest number of OTUs $(1,762)$, followed by WT and FO $(1,245)$, WT and LOCO $(1,231)$, and lastly, LOCO and LOCOAB $(1,166)$.

\section{Gut Microbiota Taxonomic Composition}

At the phylum level, Proteobacteria (21.8-57.4\%), Firmicutes (11.8-38.8\%), Bacteroidota (2.7-32.0\%), Fusobacteriota (1.3-11.1\%), and Tenericutes (0.3-52.2\%) were the core groups in all types of samples, accounting for $86.2 \%$ of the total reads (Fig. $2 \mathrm{~A}$ and $2 \mathrm{~B}$ ). The phylum Tenericutes was reclassified into the Bacilli class of Firmicutes following the SILVA database and therefore, not annotated in the 16S rRNA amplicon analysis. The WT microbiota samples were dominated by Firmicutes and Tenericutes, while in FO samples, Proteobacteria and Fusobacteria were the most abundant. Proteobacteria also dominated the LOCO microbiota, while for diet LOCOAB treatment, Bacteroidota, Proteobacteria, and Spirochaetota 
were most abundant. Experimental diets treatment reduced the abundance of Firmicutes and Tenericutes, increasing the proportion of Proteobacteria, Fusobacteria, Bacteroidota, Spirochaetota, and Campylobacterota, respectively. The microbiota of LOCO treatment showed a higher Proteobacteria, Firmicutes, and Tenericutes, and lower Bacteroidota, Fusobacteria, Spirochaetota, and Campylobacterota abundance as compared to diet FO. Treatment with diet LOCOAB lowered the abundance of Proteobacteria, Firmicutes, Tenericutes, and Fusobacteria, while Bacteroidota and Spirochaetota increased.

The Proteobacteria phylum was mainly represented by Gammaproteobacteria, predominantly from the Vibrionaceae family (Fig. S3 and S4). Fusobacteria in the FO microbiota was represented by the Fusobacteriaceae family from the Fusobacteriia class. In WT samples, the Entomoplasmatales Incertae Sedis family of Bacilli class was predominant in Firmicutes, and the Mycoplasmataceae family of Mollicutes class was most abundant among Tenericutes. Within the Bacteroidota, the Marinilabilaceae and Prolixibacteraceae families dominated the LOCOAB microbiota. At the genus level, both the $16 \mathrm{~S}$ and shotgun datasets showed the dominance of Candidatus Hepatoplasma in the WT microbiota (Fig. 2C and 2D). Compared to animals fed experimental diets, there was a reduction in Candidatus Hepatoplasma, alongside Mycoplasma, Photobacterium, Spiroplasma, Paraclostridium, Bacillus, and Hypnocyclicus in the microbiota of experimentally fed animals. In contrast, the abundance of Vibrio, Sediminispirochaeta, Sunxiuqinia, Ruegeria, Arcobacter, Malaciobacter, and Carboxylicivirga was elevated. In diet LOCO, Vibrio, Candidatus Hepatoplasma, Shewanella, and Ferrimonas showed higher abundance than diet FO, while the abundance of Sediminispirochaeta, Carboxylicivirga, Sunxiuginia, Psychrilyobacter, Arcobacter, Malaciobacter, Propionigenium, and Halarcobacter was lower. For a more indepth comparison between diet LOCO and LOCOAB, the Statistical Analysis of Metagenome Profiles analysis was carried out (Fig. S5). In LOCOAB samples, genera such as Vibrio, Candidatus Hepatoplasma, Ruegeria, Shewanella Mycoplasma, Spiroplasma, Photobacterium, and Psychrilyobacterwere reduced, while Sediminispirochaeta, Carboxylicivirga, Sunxiuqinia, and Oceanispirochaeta were elevated.

Supervised comparisons by LEfSe (LDA > 4.0) were also conducted to provide an overview of differences in taxonomic profiles for all dietary treatments (Fig. 3). The analysis identified 50 differentially abundant taxa ranging from the phylum to OTU level (12 in WT; 16 in FO; 7 in LOCO; 15 in LOCOAB).

\section{S. olivacea Gut Microbiota Functions}

A total of $84.4 \%$ of the unique gene sets from all microbiota samples were assigned into functional groups (Table S4). These genes were further annotated to 44 pathways at KEGG level 2, with the highest enrichment in global and overview maps (25.4\%), followed by carbohydrate (8.3\%) and energy $(5.2 \%)$ metabolisms (Fig. S6). The relative composition of these three categories at KEGG level 3 is illustrated in Fig. S7.

A heatmap with normalized values of functional abundance was illustrated to compare the enriched level 2 categories between the four sample groups (Fig. 4A). The WT crab microbiota showed a higher relative abundance of nucleotide metabolism, transcription, translation, folding, sorting and degradation, 
replication and repair, and signaling molecules and interaction pathways. The microbiota of crabs fed experimental diets were enriched in global and overview maps, numerous metabolisms including lipid, amino acid, other amino acids, cofactors and vitamins, terpenoids and polyketides, and biosynthesis of secondary metabolites. Among the experimental diets, LOCO resulted in enrichment in cell motility, cellular community-prokaryotes, membrane transport, signal transduction, and xenobiotics degradation and metabolism. For diet LOCOAB, highest abundance of global and overview maps, and metabolisms of major nutrients were obtained. In addition, the metagenome gene sets were also mapped against the Comprehensive Antibiotic Resistance Database (CARD) to determine the occurrence of antibiotic resistance genes (ARGs) in the S. olivacea gut microbiota (Fig. S8). We recovered ARGs from 15 main families, corresponding to seven drug mechanisms in CARD.

In relevance to the main objective of this study, a heatmap illustration to compare the KEGG Level 3 categories within lipid metabolism between dietary treatments was built (Fig. 4B). Feeding crabs with experimental diets enriched various lipid metabolism pathways, including fatty acid biosynthesis and fatty acid degradation. Compared to diet FO, pathways on fatty acid degradation, synthesis and degradation of ketone bodies, glycerophospholipid metabolism, ether lipid metabolism, linoleic acid metabolism, arachidonic acid metabolism, and biosynthesis of unsaturated fatty acids were enriched in the LOCO microbiota. Concomitantly, diet LOCOAB lowered the abundance of these functions while causing an increase in sphingolipid metabolism, steroid hormone biosynthesis, fatty acid elongation, and fatty acid biosynthesis.

Within lipid metabolism, the fatty acid biosynthesis and biosynthesis of unsaturated fatty acids are key pathways towards the biosynthesis of LC-PUFA. Numerous genes encoding for the enzymes are present in both pathways (Fig. S9 and S10). There was an increase in the abundance of several sequences assigned to the KEGG function biosynthesis of unsaturated fatty acid in diet LOCO treatment. Among these are acyl-CoA oxidase, stearoyl-CoA desaturase, and acyl-coenzyme $A$ thioesterases, which were also impeded in diet LOCOAB (Table S5). Conversely, there was higher abundance of fatty acid biosynthesis genes in the microbiota of LOCOAB treatment. The complete pathways for acetate and butyrate formation and a partial propionate pathway are present in S. olivacea gut microbiota (Fig. S11-S13). Heatmap analysis showed the LOCOAB treatment increased the function of short-chain fatty acids (SCFAs) production as compared to LOCO treatment (Fig. 4C).

In terms of bacteria taxa, genes involved in lipid metabolism were mostly contributed by Proteobacteria and represented by the families Vibrionaceae, Rhodobacteraceae, and Shewanellacea (Fig. 5A). At the genus level, lipid metabolism in WT microbiota was contributed by Vibrio, Shewanella, and Clostridium (Fig. 5B). In diet LOCO, the Ruegeria and Shewanella genera contributed higher towards lipid metabolism as compared to diet FO. While diet LOCOAB did not affect the contribution levels of these two genera, there was an increase in contribution from Vibrio, Arcobacter, Clostridium, and Bacteroides.

Several marine species, in particular Shewanella and Vibrio, can synthesize PUFA de novo through an anaerobic route, using the fatty acid synthase/polyketide synthase (FAS/PKS)-like enzyme system [40, 
41]. The gene cluster encoding this system consists of four ORFs, represented by the $p f a A B C D$ genes [42]. Since this pathway is not included in the KEGG database, we examined the $S$. olivacea gut shotgun metagenomic data sets for the diversity and distribution of the keto-acyl synthase (KS) domain harbored within the $p f a A$ homolog. The KS sequences recovered in this study form a large monophyletic clade with known KSs from Vibrio, Shewanella, and Photobacterium, showing conservation of the KS domain across species (Fig. 6). A total of $362 \mathrm{KS}$ sequences were recovered, with $36,135,127$, and 64 sequences from WT, FO, LOCO, and LOCOAB samples, respectively. Therefore, in contrast to SCFAs pathways, the inclusion of oxolinic acid impeded the PKS pathway in S. olivacea.

In hepatopancreas, the highest levels of DHA were obtained with diet FO (Table S6). Diet LOCO resulted in the deposition of a-linolenic acid, ALA and linoleic acid, LA, and also some known intermediates of the LC-PUFA biosynthesis pathway such as 18:4n-3 and 22:5n-3, which implies some degree of LC-PUFA biosynthesis. Interestingly, despite having the same lipid level and fatty acids profile, the percentage of ALA and LA were significantly higher in diet LOCOAB. Crabs fed diet FO also have the highest deposition of all three LC-PUFA in muscle tissue. There was no significant difference in LC-PUFA levels between diet LOCO and LOCOAB.

\section{Discussion}

Regardless of treatment, Proteobacteria, Firmicutes, Bacteroidota, Fusobacteriota, and Tenericutes are the main phyla in S. olivacea gut microbiota, paralleling previous studies in Scylla and Chinese mitten crab inventories [43-45]. As facultative or obligate anaerobes, the consumption of oxygen within the gut by Proteobacteria contributes to an anaerobic environment, which facilitates the proliferation of strict anaerobes [46]. Firmicutes and Bacteroidota are known to participate in carbohydrates and polysaccharides digestion [20,47]. Members of the Fusobacteriota were implicated in synthesizing vitamins and fermentation of amino acids $[48,49]$. The prevalence of Tenericutes in the vertebrate microbiota is linked to polysaccharides digestion $[50,51]$. Similar to previous findings in crabs, the main bacteria groups in wild-caught $S$. olivacea are Tenericutes and Firmicutes $[43,52]$. Candidatus Hepatoplasma, the predominant genus in the wild crabs in this study, is attributed to adaptation towards limited food availability in S. paramamosain [43]. In the isopod intestinal tract, this genus conferred a higher host survival rate during food scarcity [53]. We also observed lower proportions of Firmicutes, Tenericutes, and Candidatus Hepatoplasma in crabs maintained by the experimental diets. From a dietary perspective, this alteration could be due to a higher intake of dietary lipid as compared to the wild-caught crabs.

The highest bacteria diversity index observed in the microbiota of treatment FO associates high dietary n3 LC-PUFA intake with gut microbiota diversity. The microbiota community in $S$. paramamosain and $P$. vannamei were more diverse with FO-based diet than soybean oil-based diet treatment $[20,44]$. In the fish intestine, dietary n-3 LC-PUFA was reported to reverse microbial dysbiosis by inhibiting pathogenic bacteria, allowing the proliferation of healthy bacteria [54-56]. Higher gut microbiota diversity was also reported in transgenic animals with high tissue n-3 PUFA content phenotype $[57,58]$. A recent study on $S$. 
serrata microbiota diversity reported no significant difference between wild-caught and farmed crabs [52]. However, this could be due to the use of trash fish in farms, which may have a similar lipid profile with the natural prey consumed by wild crabs.

Compared to diet FO, there was an apparent spike in the abundance of Proteobacteria, mainly represented by the Vibrio genus, in the microbiota of crabs fed diet LOCO. In a marine fish fed vegetable oil (VO)-rich diet, a higher proportion of Vibrio in the intestine microbiota was reported [55]. The gut microbiota profiles of several farmed decapod species also highlighted the dominance of this genus [59]. Although Vibrio can be pathogenic, gut Vibrio can confer beneficial effects on the host through the secretion of beneficial digestive enzymes [60]. In swimming crabs, high dietary lipid levels also induced the relative abundance of Proteobacteria and Vibrio [61]. It is worth noting that crab-fed diet LOCO also displayed a higher abundance of another Proteobacteria representative, the Shewanella genus. Pertinent to our main objective, these two genera are known to possess PUFA-biosynthesis capacity [40,62-64]. Therefore, we showed for the first time the increased abundance of two known PUFA biosynthesis bacteria genera in the microbiota of Scylla mud crabs fed a diet with limited LC-PUFA but higher availability of C18 PUFA, which are substrates for LC-PUFA biosynthesis.

In addition to taxonomy profiling of bacterial communities, we assessed major shifts in the function of crabs microbiota of different dietary treatments. The prominence of global and overview maps, carbohydrate, amino acid, and carbohydrate metabolisms in our samples paralleling the observation in white shrimp Litopenaeus vannamei [59]. We also observed a higher abundance of sequences related to genetic information processing and nucleotide metabolism in wild-caught crabs compared to those that underwent the feeding trial, which could indicate higher stress conditions in natural habitats. In tandem, the enrichment of global and overview maps and the pathways for various metabolisms such as lipid, amino acid, other amino acids, cofactors and vitamins, terpenoids and polyketides, and biosynthesis of secondary metabolites in experimentally fed crabs suggested optimal provision of dietary energy, protein, and lipid. The higher dietary lipid intake in experimentally fed crab provides the substrates for higher lipid metabolic activities, which are crucial to supply hydrophobic components of the cell membrane and the generation of cellular energy $[65,66]$.

We demonstrate that diet LOCO resulted in the highest abundance of genes encoding for fatty acid biosynthesis and biosynthesis of unsaturated fatty acids within the Scylla gut microbiota. This trend is correlated with an increase in contribution towards lipid metabolism by Shewanella and Ruegeria. The biosynthesis of fatty acids from acetyl-CoA through the type I or type II FAS system will be preceded by a series of condensation, reduction, dehydration, and reduction reactions, leading to the formation of longchain fatty acids [63]. Besides the two above-mentioned pathways, our results showed an increase in pathways related to SCFAs production in crabs fed diet LOCO as compared to diet FO treatment. In the gut, non-digestible carbohydrates are fermented into SCFAs, primarily acetate, propionate, and butyrate. These SCFAs can act as precursors for hepatic desaturation and elongation activities for further conversion to LC-PUFA [67]. Significant hits matching the KS domain were also obtained from FO or LOCO-fed S. olivacea metagenome datasets, indicating enrichment of the PKS-like pathway. This 
alternative LC-PUFA biosynthesis pathway was initially discovered in marine microorganisms and subsequently reported to be widespread in prokaryotes from various niches, including intestinal microbiota $[40,68,69]$. The number of KS sequences detected in our samples is noticeably higher than those obtained from environmental metagenomes [70], which supports the speculation that aquatic animals' guts are hotspots for LC-PUFA biosynthesis. The higher abundance of genes encodings for the PKS-pathway functions in diet LOCO treatment is associated with the higher composition of Shewanella and Vibrio, two known genera with the capacity for EPA and DHA biosynthesis through the PKS system $[40,41]$. Overall, our results suggest elevated abundance in genes related to various pathways for LCPUFA biosynthesis in S. olivacea fed diet LOCO.

Oxolinic acid, a broad-spectrum antibiotic commonly used in aquaculture [71], was utilized in this study as a non-invasive and unbiased approach to hypothetically impede the proliferation and function of gut bacterial with a role in LC-PUFA biosynthesis. A similar approach was used to limit the contribution of microbiota bacterial groups with speculated roles in lipid metabolism $[67,69]$. We observed a reduction in gut microbiota diversity in S. olivacea fed diet LOCOAB, consistent with previous findings [69, 72]. There was a reduction in the proportion of Proteobacteria, caused mainly by a decrease in Vibrio and Shewanella. In contrast, oxolinic acid promoted the proliferation of the phyla Spirochaetota and Bacteroidota, which were represented by Sediminispirochaeta, Carboxylicivirga, and Sunxiuginia. Function wise, the inclusion of oxolinic acid impeded the recovery of gene sets from several lipid metabolism pathways, including biosynthesis of unsaturated fatty acids and PKS pathway. However, higher abundance of sequences for SCFAs synthesis, primarily acetate and butyrate, were obtained. This increase could be associated with the abundance of Bacteroidota, a known acetate producer in the microbiota [73].

In Chinese mitten crab, the gut microbial community influences the host fatty acid composition [74]. Elsewhere, PUFA-biosynthesizing zooxanthellae symbionts were shown to affect the LC-PUFA composition of their respective coral hosts $[75,76]$. Therefore, we relate the compounding changes in structure and function of $S$. olivacea microbiota fed the different experimental diets to their respective hepatopancreas and muscle PUFA composition. We found lower LC-PUFA content in hepatopancreas and muscle of crab fed diet LOCO or LOCOAB as compared to diet FO. This reiterates previous findings on the fatty acid profiles of mud crabs fed VO-based diets $[77,78]$. Therefore, the LC-PUFA biosynthesis activities driven by the in vivo enzymatic capacity of $S$. olivacea or gut bacterial communities in the VO-based diets are unable to produce tissue deposition levels of LC-PUFA which are comparable to diet FO. Despite metagenome datasets showing a reduction in the composition of Vibrio and Shewanella, and several unsaturated fatty acids biosynthesis activities in the microbiota of crab fed diet LOCOAB, there was no significant reduction in levels of muscle LC-PUFA when compared to the values of LOCO treatment. As aforementioned, there was an increase in the function of SCFAs production within the microbiota of LOCOAB crab, which could be a source of LC-PUFA for the host. Secondly, although the composition of Proteobacteria and Vibrio were reduced in the microbiota of LOCOAB treatment, these two groups still occupied a significant proportion of the overall microbiota diversity and therefore, might still contribute in terms of LC-PUFA biosynthesis. In sesarmid crabs, antibiotic treatment did not result in a significantly 
lower level of DHA, presumably due to incomplete removal of gut bacteria [69]. Additionally, the possibility of representatives from the phylum Bacteroidota contributing to LC-PUFA biosynthesis could not be ruled out. The persistence of EPA and ARA in the muscle tissues of LOCOAB crabs could also be due to the lower utilization of these PUFAs as substrates for eicosanoid production. This reason is partially supported by the observed decrease in arachidonic acid metabolism in the metagenome dataset from LOCOAB. Lastly, the lack of molting observed in LOCOAB crabs during the whole feeding trial could also mean the conservation of LC-PUFA in tissues [79].

\section{Conclusion}

Overall, our study shed light on the LC-PUFA biosynthesis activities within the gut microbiota of $S$. olivacea. The microbiota of crabs with high C18 PUFA and limited LC-PUFA intake showed an increase in Vibrio and Shewanella, which are known for LC-PUFA biosynthesis, alongside an increase in various lipid metabolism pathways. Although the use of an antibiotic-supplemented experimental feed did not lower the deposition of LC-PUFA in crab muscle, changes within the bacterial taxa groups and several lipid functions suggest some degree of persistency in LC-PUFA biosynthesis activities. Taken together, these findings showed the effect of dietary PUFA intake on the microbiota composition, accentuating a contribution by gut microbiota in LC-PUFA biosynthesis activities.

\section{Declarations}

\section{Acknowledgments}

This study was supported by a Universiti Sains Malaysia (USM) short-term research grant (304/PCCB/6315222). Dr. Khor Waiho is an adjunct academic fellow at the Center for Chemical Biology, USM.

\section{Conflict of Interest}

The authors declare that they have no conflict of interest.

\section{Data availability}

The raw sequence data have been deposited in NCBI Sequence Read Archive under BioProject accession PRJNA737307. The metagenome assemblies have been deposited in NCBI under the same BioProject accession.

\section{References}

1. Swanson D et al (2012) Omega-3 fatty acids EPA and DHA: health benefits throughout life. Adv Nutr $3: 1-7$ 
2. Burdge GC (2006) Metabolism of a-linolenic acid in humans. Prostaglandins Leukot Essent Fatty Acids 75:161-168

3. Arts MT et al (2001) "Essential fatty acids" in aquatic ecosystems: a crucial link between diet and human health and evolution. Can J Fish Aquat Sci 58:122-137

4. Sprecher $H$ (2000) Metabolism of highly unsaturated $n-3$ and $n-6$ fatty acids. Biochim Biophys Acta 1486:219-231

5. Twining CW et al (2021) The evolutionary ecology of fatty-acid variation: Implications for consumer adaptation and diversification. Ecol Lett 24:1709-1731

6. Viña-Trillos N, Urzúa Á (2021) Comparison of lipids and fatty acids among tissues of two semiterrestrial crabs reveals ecophysiological adaptations in changing coastal environments. Comp Biochem Physiol Part A Mol Integr Physiol 259:111012

7. Matsushita $Y$ et al (2020) Flatfishes colonised freshwater environments by acquisition of various DHA biosynthetic pathways. Commun Biol 3:516

8. Kabeya $\mathrm{N}$ et al (2018) Genes for de novo biosynthesis of omega-3 polyunsaturated fatty acids are widespread in animals. Sci Adv 4:eaar6849-eaar6849

9. Monroig Ó, Kabeya N (2018) Desaturases and elongases involved in polyunsaturated fatty acid biosynthesis in aquatic invertebrates: a comprehensive review. Fisheries Sci 84:911-928

10. Kabeya $\mathrm{N}$ et al (2021) A complete enzymatic capacity for biosynthesis of docosahexaenoic acid (DHA, 22: 6n-3) exists in the marine Harpacticoida copepod. Open Biol 11:200402

11. Ting SY et al (2020) Two elongases, Elovl4 and Elovl6, fulfill the elongation routes of the LC-PUFA biosynthesis pathway in the orange mud crab (Scylla olivacea). J Agric Food Chem 68:4116-4130

12. Mah MQ et al (2019) Molecular cloning, phylogenetic analysis and functional characterisation of an Elovl7-like elongase from a marine crustacean, the orange mud crab (Scylla olivacea). Comp Biochem Physiol B Biochem Mol Biol 232:60-71

13. Sun $P$ et al (2020) Cloning and functional characterization of an elovl4-like gene involved in the biosynthesis of long-chain polyunsaturated fatty acids in the swimming crab Portunus trituberculatus. Comp Biochem Physiol B Biochem Mol Biol 242:110408

14. Monroig Ó, Kabeya N (2018) Desaturases and elongases involved in polyunsaturated fatty acid biosynthesis in aquatic invertebrates: a comprehensive review. Fisheries Sci 84:911-928

15. Keenan CP et al (1998) A revision of the genus Scylla de Haan, 1833 (Crustacea: Decapoda : Brachyura : Portunidae). Raffles B Zool 46:217-245

16. Syafaat MN et al (2021) A review of the nursery culture of mud crabs, genus Scylla: Current progress and future directions. Animals 11: 2034

17. Harris JM (1993) The presence, nature, and role of gut microflora in aquatic invertebrates: A synthesis. Microb Ecol 25:195-231

18. Bäckhed $\mathrm{F}$ et al (2004) The gut microbiota as an environmental factor that regulates fat storage. Proc Natl Acad Sci U S A 101:15718-15723 
19. Semova I et al (2012) Microbiota regulate intestinal absorption and metabolism of fatty acids in the zebrafish. Cell Host Microbe 12:277-288

20. Zhang $M$ et al (2014) Characterization of the intestinal microbiota in Pacific white shrimp, Litopenaeus vannamei, fed diets with different lipid sources. Aquaculture 434:449-455

21. Sun $P$ et al (2018) Dietary lipid levels could improve growth and intestinal microbiota of juvenile swimming crab, Portunus trituberculatus. Aquaculture 490:208-216

22. Caesar $\mathrm{R}$ et al (2016) Interaction between dietary lipids and gut microbiota regulates hepatic cholesterol metabolism. J Lipid Res 57:474-481

23. Dailey FE et al (2016) The microbiota of freshwater fish and freshwater niches contain omega-3 fatty acid-producing Shewanella species. Appl Environ Microbiol 82:218-231

24. Bui THH, Lee SY (2015) Potential contributions of gut microbiota to the nutrition of the detritivorous sesarmid crab Parasesarma erythodactyla. Mar Biol 162:1969-1981

25. Tajima $\mathrm{K}$ et al (2001) Diet-dependent shifts in the bacterial population of the rumen revealed with real-time PCR. Appl Environ Microbiol 67:2766-2774

26. Lau NS et al (2018) Diversity and coding potential of the microbiota in the photic and aphotic zones of tropical man-made lake with intensive aquaculture activities: A case study on Temengor Lake, Malaysia. Microb Ecol 78:20-32

27. Caporaso JG et al (2010) QIIME allows analysis of high-throughput community sequencing data. Nat Methods 7:335-336

28. Edgar RC et al (2011) UCHIME improves sensitivity and speed of chimera detection. Bioinformatics 27:2194-2200

29. Edgar RC (2013) UPARSE: highly accurate OTU sequences from microbial amplicon reads. Nat Methods 10:996-998

30. Quast $C$ et al (2013) The SILVA ribosomal RNA gene database project: improved data processing and web-based tools. Nucleic Acids Res 41:D590-D596

31. Cole JR et al (2009) The Ribosomal Database Project: improved alignments and new tools for rRNA analysis. Nucleic Acids Res 37:D141-D145

32. Segata $\mathrm{N}$ et al (2011) Metagenomic biomarker discovery and explanation. Genome Biol 12:R60-R60

33. Wood DE et al (2019) Improved metagenomic analysis with Kraken 2. Genome Biol 20:257

34. Li D et al (2015) MEGAHIT: an ultra-fast single-node solution for large and complex metagenomics assembly via succinct de Bruijn graph. Bioinformatics 31:1674-1676

35. Tang B et al (2020) Chromosome-level genome assembly reveals the unique genome evolution of the swimming crab (Portunus trituberculatus). GigaScience 9:giz161

36. Hyatt $D$ et al (2012) Gene and translation initiation site prediction in metagenomic sequences. Bioinformatics 28:2223-2230

37. Fu L et al (2012) CD-HIT: accelerated for clustering the next-generation sequencing data. Bioinformatics 28:3150-3152 
38. Alcock BP et al (2020) CARD 2020: antibiotic resistome surveillance with the comprehensive antibiotic resistance database. Nucleic Acids Res 48:D517-D525

39. Stamatakis A (2014) RAxML version 8: a tool for phylogenetic analysis and post-analysis of large phylogenies. Bioinformatics 30:1312-1313

40. Metz JG et al (2001) Production of polyunsaturated fatty acids by polyketide synthases in both prokaryotes and eukaryotes. Science 293:290-293

41. Nichols DS, McMeekin TA (2002) Biomarker techniques to screen for bacteria that produce polyunsaturated fatty acids. J Microbiol Methods 48:161-170

42. Allen EE, Bartlett DH (2002) Structure and regulation of the omega-3 polyunsaturated fatty acid synthase genes from the deep-sea bacterium Photobacterium profundum strain SS9. Microbiology 148:1903-1913

43. Wei H et al (2019) High-throughput sequencing reveals the core gut microbiota of the mud crab (Scylla paramamosain) in different coastal regions of southern China. BMC Genom 20:829-829

44. Luo J et al (2021) Dietary soybean oil aggravates the adverse effects of low salinity on intestinal health in juvenile mud crab Scylla paramamosain. Ecotoxicol Environ Saf 213:112004

45. Wang $C$ et al (2019) Change in the intestinal bacterial community structure associated with environmental microorganisms during the growth of Eriocheir sinensis. Microbiology Open 8:e00727

46. Shin N-R et al (2015) Proteobacteria: microbial signature of dysbiosis in gut microbiota. Trends Biotechnol 33:496-503

47. Ghanbari $\mathrm{M}$ et al (2015) A new view of the fish gut microbiome: Advances from next-generation sequencing. Aquaculture 448:464-475

48. Rowland I et al (2018) Gut microbiota functions: metabolism of nutrients and other food components. Eur J Nutr 57:1-24

49. Dai ZL et al (2011) Amino acid metabolism in intestinal bacteria: links between gut ecology and host health. Front Biosci 16:1768-1786

50. Mente E et al (2018) Core versus diet-associated and postprandial bacterial communities of the rainbow trout (Oncorhynchus mykiss) midgut and faeces. Biol Open 7:bio034397

51. Yan X et al (2016) Microflora Disturbance during Progression of Glucose Intolerance and Effect of Sitagliptin: An Animal Study. J Diabetes Res 2016: 2093171

52. Apine E et al (2021) Comparative analysis of the intestinal bacterial communities in mud crab Scylla serrata in South India. Microbiology Open 10:e1179

53. Fraune S, Zimmer M (2008) Host-specificity of environmentally transmitted Mycoplasma-like isopod symbionts. Environ Microbiol 10:2497-2504

54. Ring $\varnothing \mathrm{E}$ et al (1998) The effect of dietary fatty acids on lactic acid bacteria associated with the epithelial mucosa and from faecalia of Arctic charr, Salvelinus alpinus (L.). J Appl Microbiol 85:855864 
55. You C et al (2019) Effects of dietary lipid sources on the intestinal microbiome and health of golden pompano (Trachinotus ovatus). Fish Shellfish Immunol 89:187-197

56. You C et al (2021) Evaluation of different dietary n-3 Ic-pufa on the growth, intestinal health and microbiota profile of golden pompano (Trachinotus ovatus). Aquac Nutr 27:953-965

57. Bidu $C$ et al (2018) The transplantation of $\omega 3$ PUFA-altered gut microbiota of fat-1 mice to wild-type littermates prevents obesity and associated metabolic disorders. Diabetes 67:1512-1523

58. Chiang KY et al (2020) Dual expression of transgenic delta- 5 and delta- 6 desaturase in tilapia alters gut microbiota and enhances resistance to Vibrio vulnificus infection. PLoS One 15:e0236601

59. Gao S et al (2019) Metagenomic insights into the structure and function of intestinal microbiota of the farmed Pacific white shrimp (Litopenaeus vannamei). Aquaculture 499:109-118

60. Ray AK et al (2012) Enzyme-producing bacteria isolated from fish gut: a review. Aquac Nutr 18:465492

61. Sun P et al (2018) Dietary lipid levels could improve growth and intestinal microbiota of juvenile swimming crab, Portunus trituberculatus. Aquaculture 490:208-216

62. Fang $\mathrm{J}$ et al (2004) Biosynthesis and dietary uptake of polyunsaturated fatty acids by piezophilic bacteria. Comp Biochem Physiol B Biochem Mol Biol 137:455-461

63. Russell NJ, Nichols DS (1999) Polyunsaturated fatty acids in marine bacteria--a dogma rewritten. Microbiology 145(Pt 4):767-779

64. Ring $\varnothing \mathrm{E}$ et al (1992) Production of eicosapentaenoic acid by freshwater Vibrio. Lipids 27:564-566

65. Parsons JB, Rock CO (2013) Bacterial lipids: Metabolism and membrane homeostasis. Prog Lipid Res 52:249-276

66. Jimenez-Diaz L et al (2017) Pathways for the degradation of fatty acids in bacteria. In: Rojo F (ed) Aerobic utilization of hydrocarbons, oils and lipids. Springer International Publishing, Cham, pp 1-23

67. Kindt A et al (2018) The gut microbiota promotes hepatic fatty acid desaturation and elongation in mice. Nat Commun 9:3760

68. Yazawa K (1996) Production of eicosapentaenoic acid from marine bacteria. Lipids 31 Suppl: S297300

69. Bui THH, Lee SY (2015) Potential contributions of gut microbiota to the nutrition of the detritivorous sesarmid crab Parasesarma erythodactyla. Mar Biol 162:1969-1981

70. Shulse CN, Allen EE (2011) Diversity and distribution of microbial long-chain fatty acid biosynthetic genes in the marine environment. Environ Microbiol 13:684-695

71. Lunestad BT, Samuelsen $O$ (2008) 4 - Veterinary drug use in aquaculture. In: Lie $\varnothing$ (ed) Improving Farmed Fish Quality and Safety. CRC Press, Boca Raton, pp 97-127

72. Wang E et al (2019) Consumption of florfenicol-medicated feed alters the composition of the channel catfish intestinal microbiota including enriching the relative abundance of opportunistic pathogens. Aquaculture 501:111-118 
73. Parada Venegas D et al (2019) Short chain fatty acids (SCFAs)-mediated gut epithelial and immune regulation and its relevance for inflammatory bowel diseases. Front Immunol 10:277

74. Su S et al (2020) Relationship between the fatty acid profiles and gut bacterial communities of the Chinese mitten crab (Eriocheir sinensis) from ecologically different habitats. Front Microbiol 11:565267

75. Papina M et al (2003) Symbiotic zooxanthellae provide the host-coral Montipora digitata with polyunsaturated fatty acids. Comp Biochem Physiol B Biochem Mol Biol 135:533-537

76. Garrett TA et al (2013) Comparative lipid profiling of the cnidarian Aiptasia pallida and its dinoflagellate symbiont. PLoS One 8:e57975

77. Ting SY et al (2020) Two elongases, Elovl4 and Elovl6, fulfill the elongation routes of the LC-PUFA biosynthesis pathway in the orange mud crab (Scylla olivacea). J Agric Food Chem 68:4116-4130

78. Unnikrishnan $U$ et al (2010) Efficacy of various lipid supplements in formulated pellet diets for juvenile Scylla serrata. Aquac Res 41:1498-1513

79. Holme M-H et al (2007) Survival, development and growth response of mud crab, Scylla serrata, megalopae fed semi-purified diets containing various fish oil:corn oil ratios. Aquaculture 269:427435

\section{Figures}


A

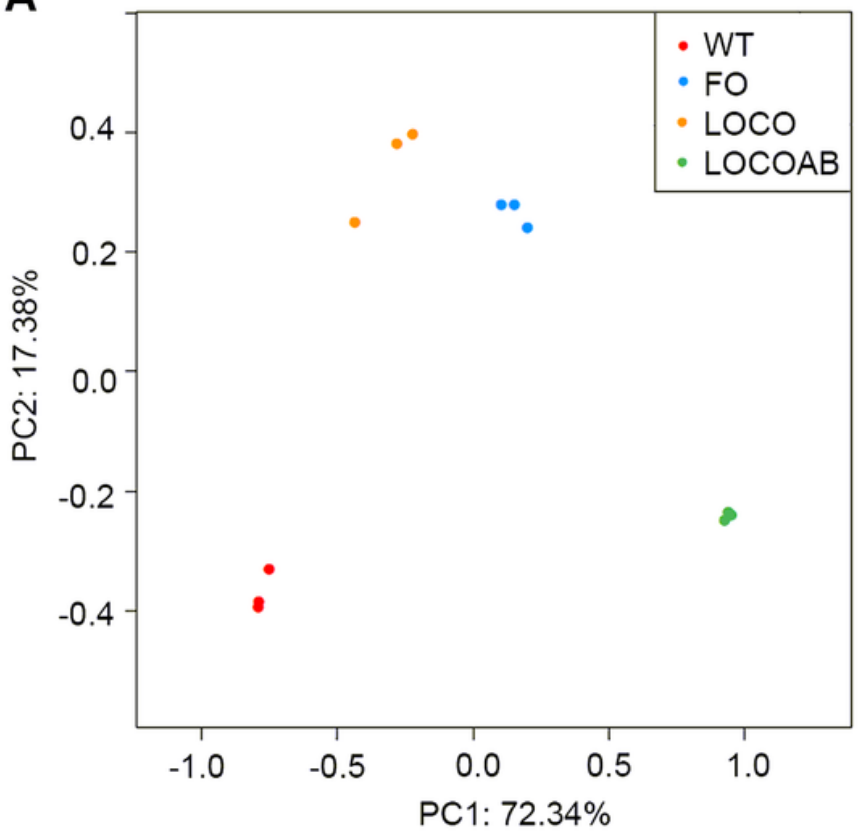

C

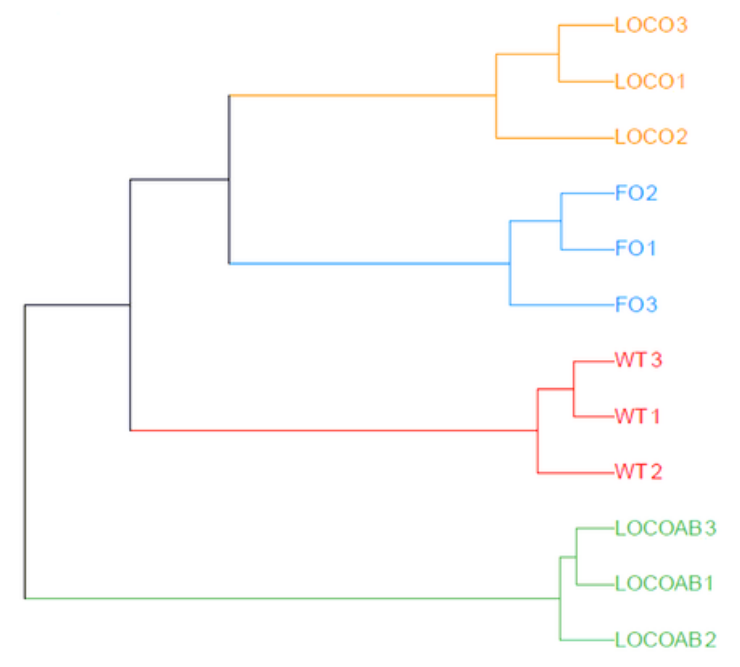

$0 . \breve{01}$
B

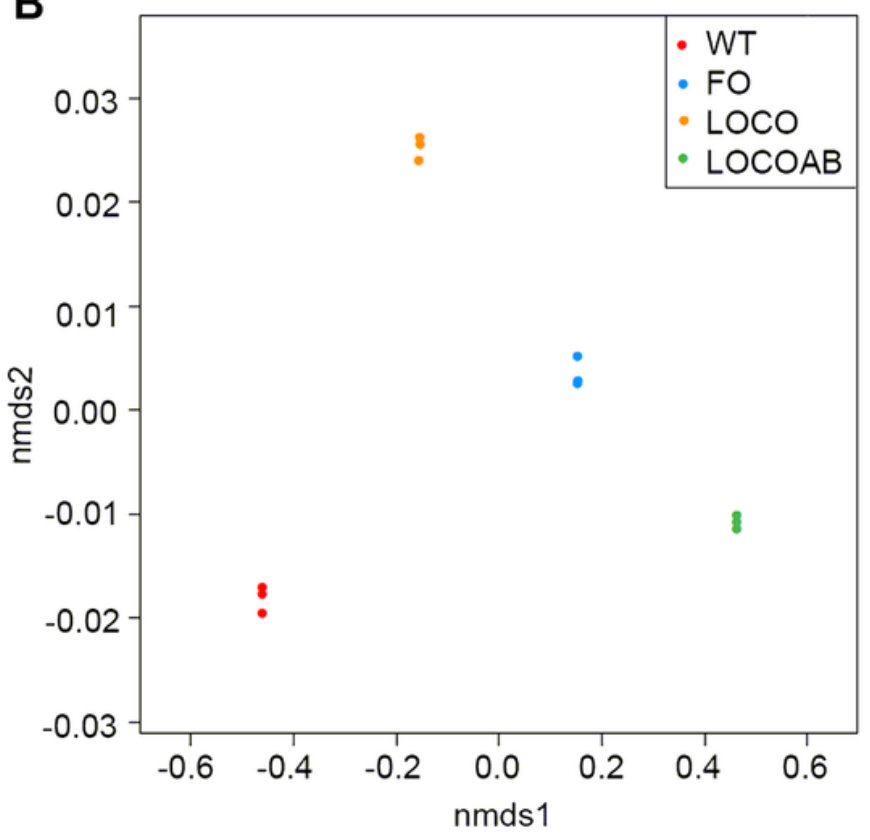

D

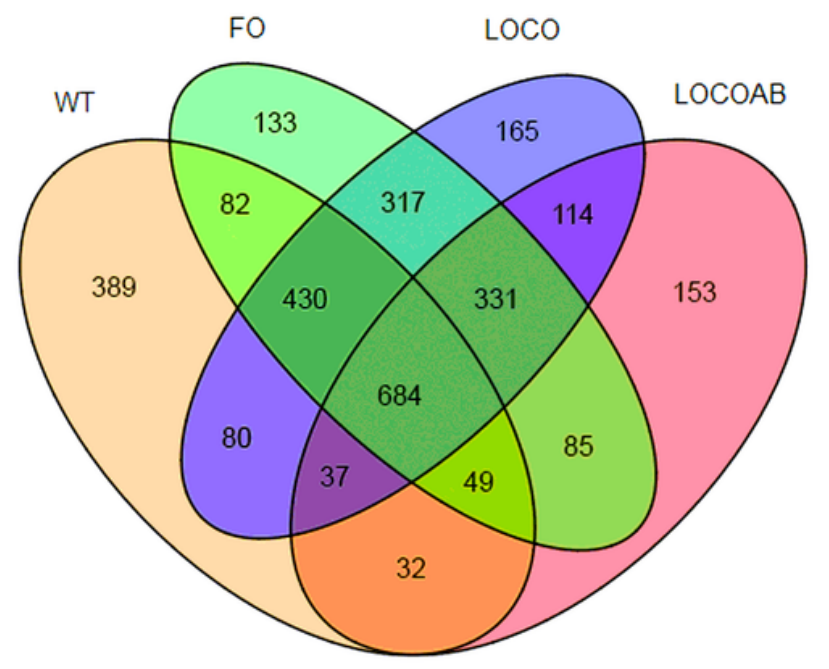

\section{Figure 1}

The bacterial community composition and relatedness of $S$. olivacea gut microbiota from wild crabs (WT); fish oil (FO); linseed oil and corn oil (LOCO); and linseed oil, corn oil and oxolinic acid (LOCOAB) samples. (A) Principal coordinate of the weighted UniFrac distance and (B) non-metric multidimensional scaling of the Bray-Curtis distance analyses of the OTUs. (C) Unweighted pair group method with arithmetic mean clustering tree of the OTUs. (D) Venn diagram showing the number of shared and unique OTUs among different crab samples. 
A

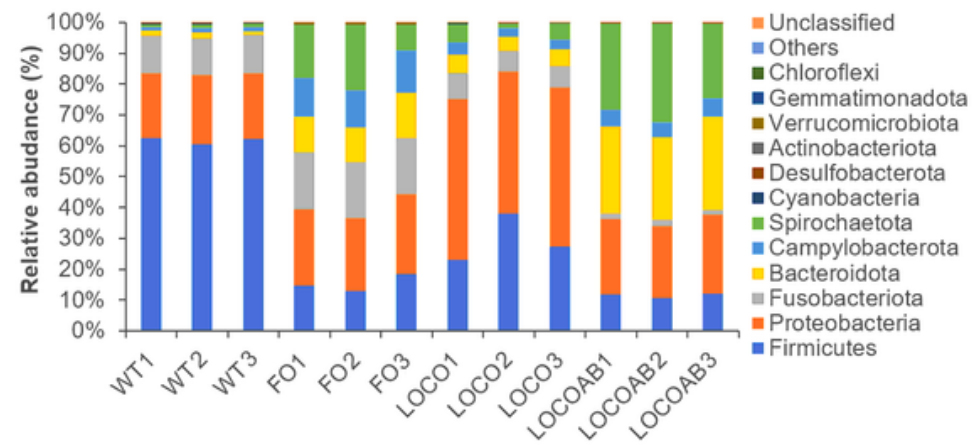

B

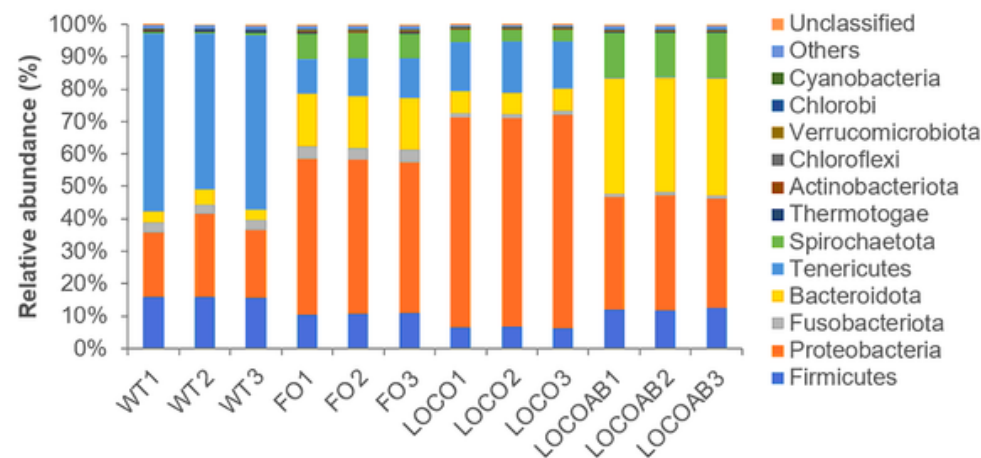

C

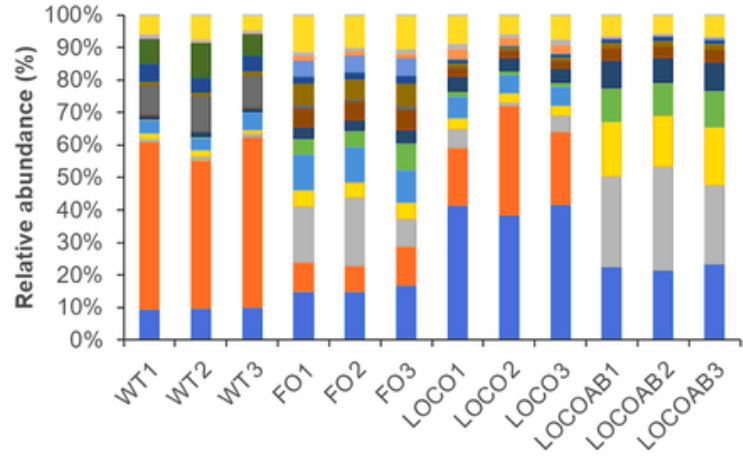

Unclassified

= Others

= Shewanella

- Ferrimonas

- Psychrilyobacter

- Paraclostridium

- Hypnocyclicus

- Halarcobacter

- Photobacterium

- Malaciobacter

- Mycoplasmataceae uncultured

- Sunxiuqinia

- Propionigenium

- Carboxylicivirga

Sediminispirochaeta

- Candidatus Hepatoplasma

- Vibrio

D

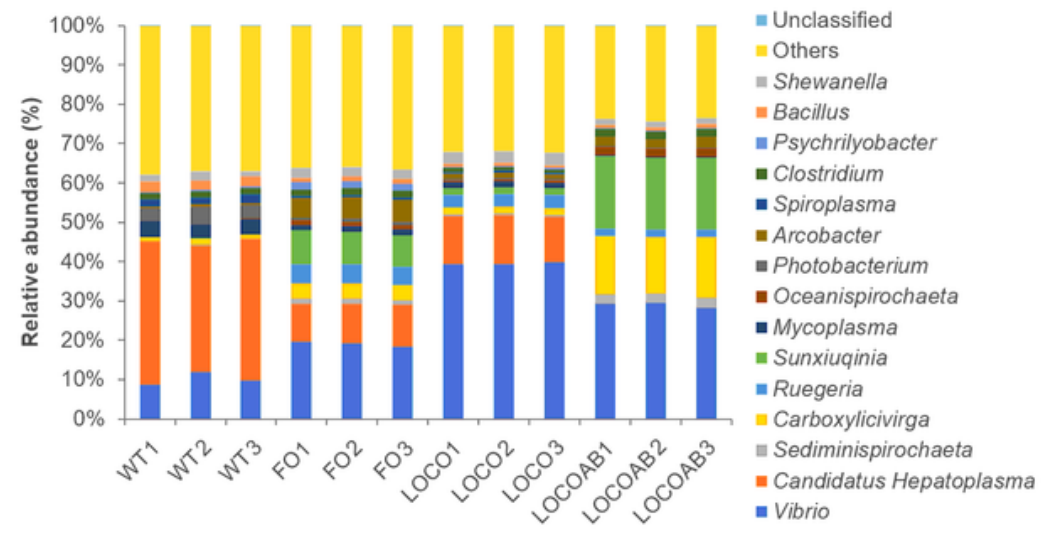

Figure 2

The composition of gut microbiota in S. olivacea from wild crabs (WT); fish oil (FO); linseed oil and corn oil (LOCO); and linseed oil, corn oil and oxolinic acid (LOCOAB) samples. Phyla analyzed by (A) 16S rRNA amplicon and (B) shotgun metagenomic sequencing. Genera analyzed by (C) $16 \mathrm{~S}$ amplicon and (D) shotgun sequencing. Phyla with percentage value of less than $0.1 \%$ are included in "Others", and only the top 15 highly abundant genera are shown. 


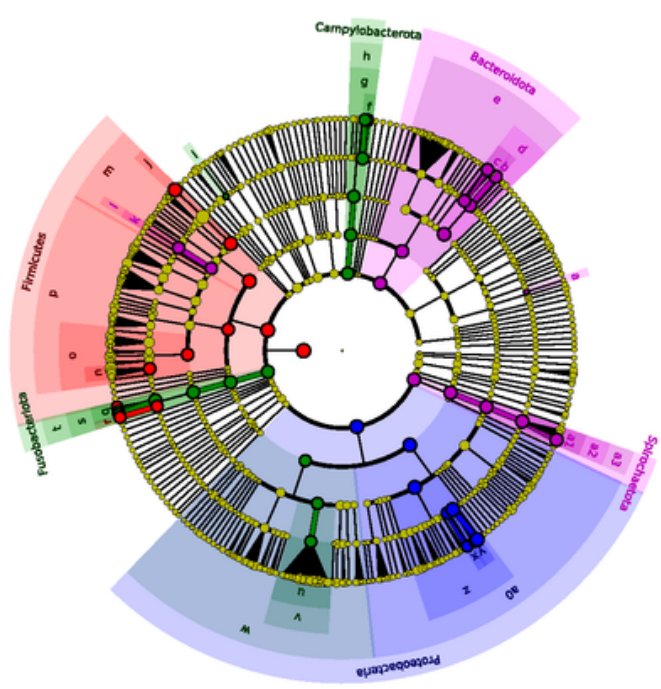

D: Microtrichacese

c: Probibactoracen

:- Bactorolidia

g. Campylobacterales

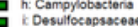

k: Mycopiassmatacesese

EMycoplasmotales

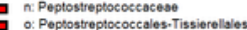

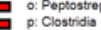

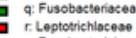

s. Fuscobcteriation

v. Rhodobactioracen

w. Alphaproteobasteris

2 Emerobactionales

a1: Spirochatacteae

a2: Spirochsotalas

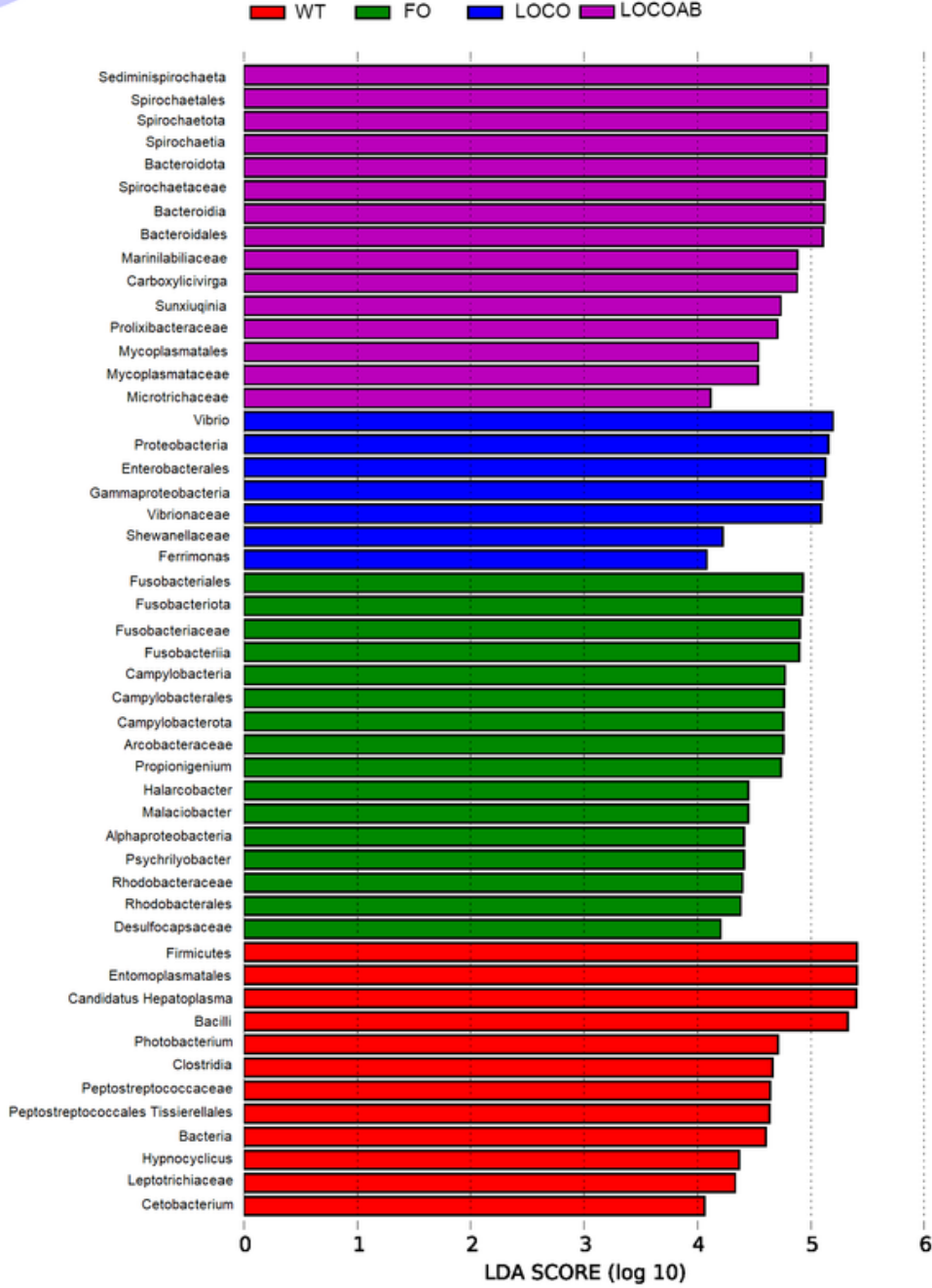

Figure 3

The linear discriminant analysis EffectSize analysis displaying the differences in gut microbiota of $S$. olivacea. (Red) taxa enriched in wild crabs (WT); (green) taxa enriched in fish oil (FO); (blue) taxa enriched in linseed oil and corn oil (LOCO); and (purple) taxa enriched in linseed oil, corn oil and oxolinic acid (LOCOAB) microbiota. 
A
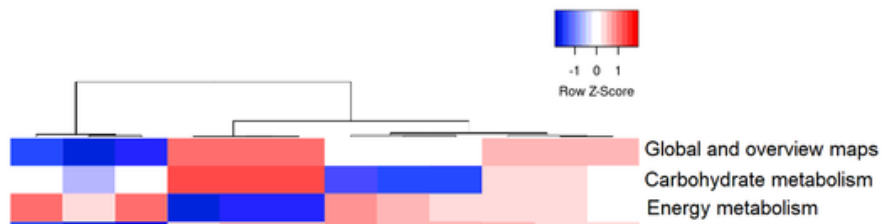

tabolism

Lipid metabolism

Nucleotide metabolism

Amino acid metabolism

Metabolism of other amino acids

Glycan biosynthesis and metabolism

Metabolism of cofactors and vitamins

Metabolism of terpenoids and polyketides

Biosythesis of other secondary metabolites

Xenobiotics biodegradation and metabolism

Transcription

Translation

Folding, sorting and degradation

Replication and repair

Membrane transport

Signal transduction

Signaling molecules and interaction

Transport and catabolism

Cell growth and death

Cellular community- prokaryotes Cell motility

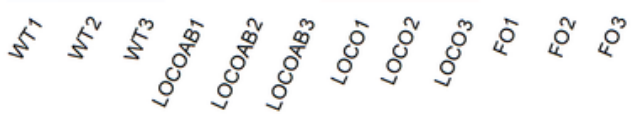

B

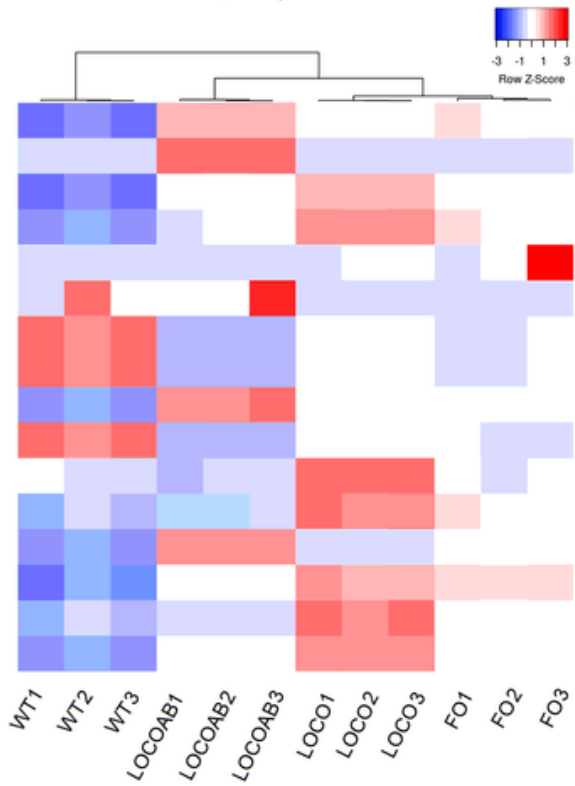

C

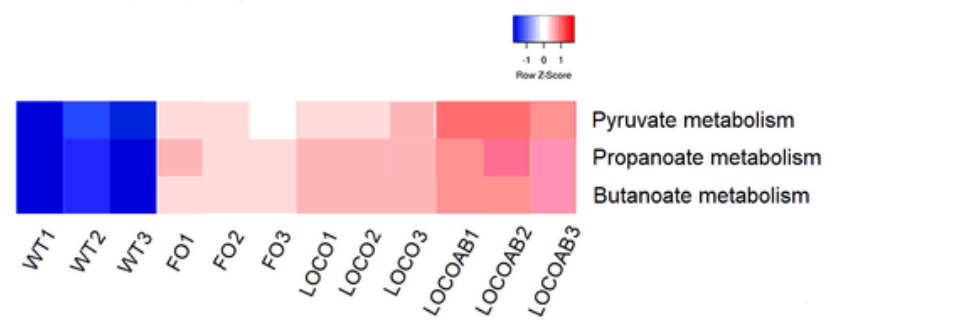

Fatty acid biosynthesis

Fatty acid elongation

Fatty acid degradation

Synthesis and degradation of ketone bodies

Cutin, suberin and wax biosynthesis

Steroid biosynthesis

Primary bile acid biosynthesis

Secondary bile acid biosynthesis

Steroid hormone biosynthesis

Glycerolipid metabolism

Glycerophospholipid metabolism

Ether lipid metabolism

Sphingolipid metabolism

Arachidonic acid metabolism

Linoleic acid metabolism

Biosynthesis of unsaturated fatty acids 


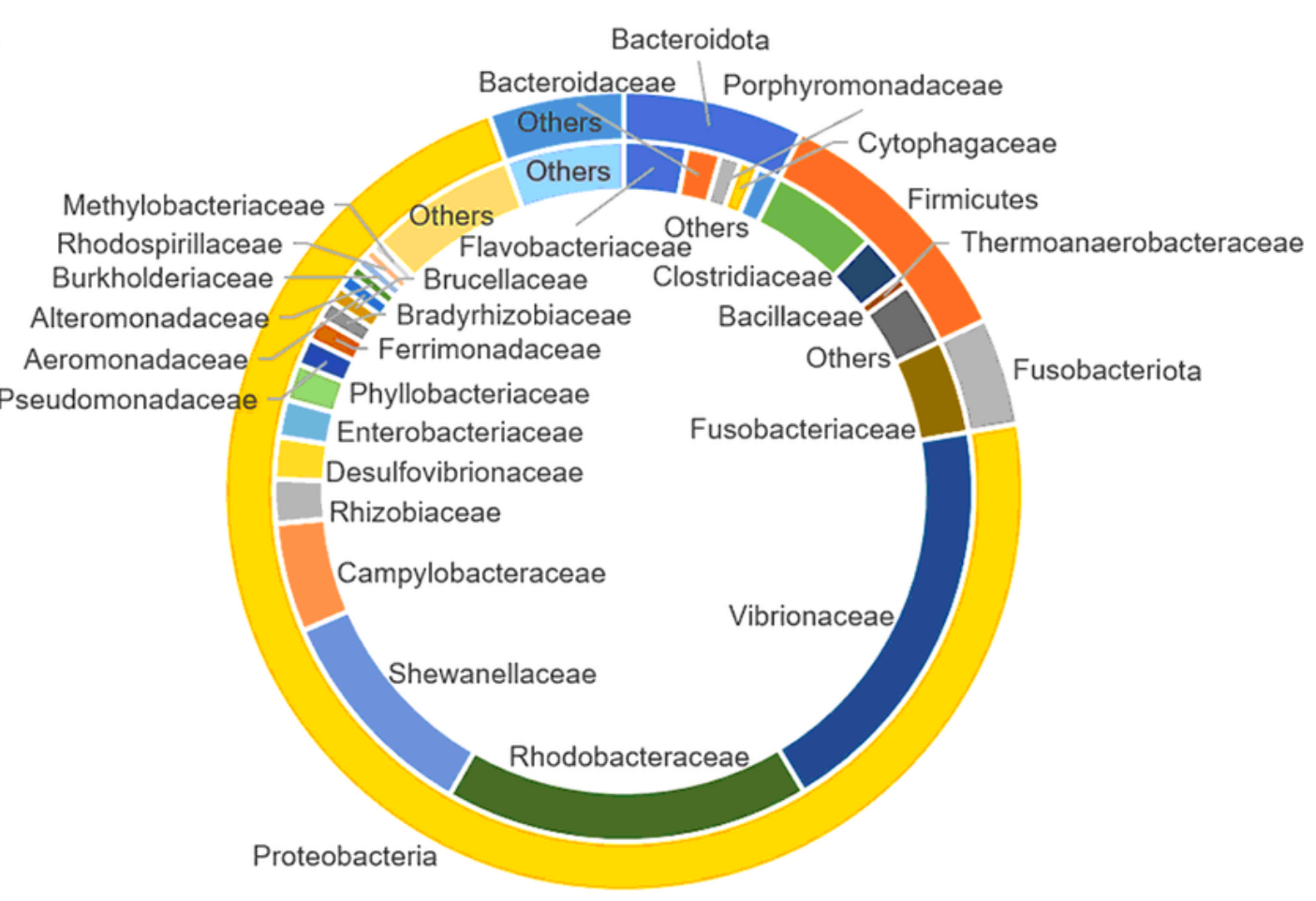

B

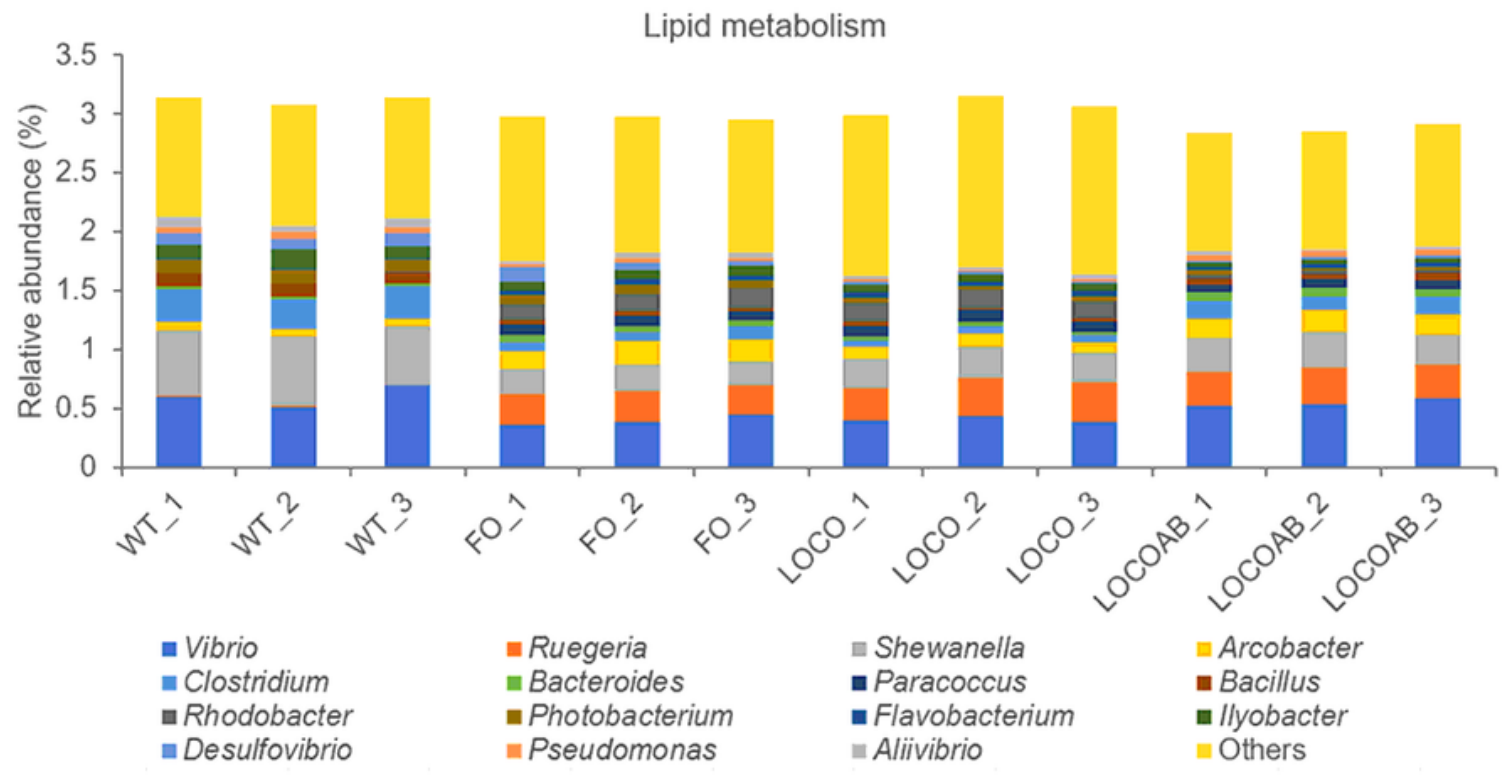

Figure 5

The contribution of $S$. olivacea gut microbiota to KEGG level 2 lipid metabolism at (A) phyla, families and (B) genera levels. The phyla and families distributions were determined based on average from 12 metagenomes. The relative abundance of genera was determined by dividing the abundance of genera annotated to lipid metabolism by the total bacterial abundance. 


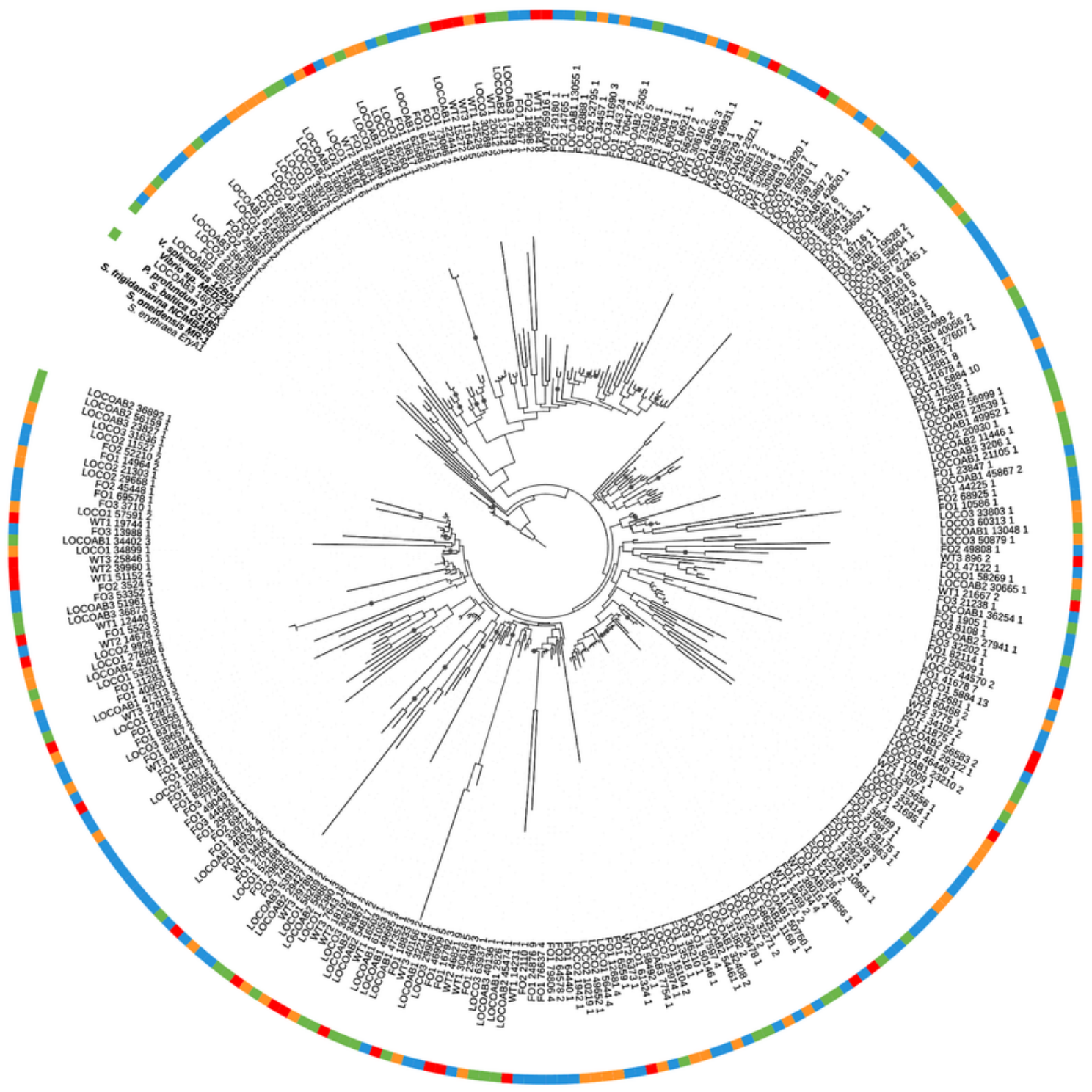

\section{Figure 6}

Maximum likelihood phylogenetic tree of keto-acyl synthase (KS) protein sequences inferred using RAxML (GAMMA+VT model, 1000 bootstraps). KSs were aligned using MUSCLE, and the alignments were trimmed using trimAl (v1.2). The best model for the amino acid alignment was evaluated using ProTest (v3.4.2) based on Akaike criterion. The Saccharopolyspora erythraea EryAI KS sequence was used as the outgroup. The phylogenetic tree was edited using the Interactive Tree of Life 
(http://itol.embl.de). Sequences from WT, FO, LOCO and LOCOAB samples are indicated by red, blue, orange, and green strips, respectively. Reference strain sequences are shown in bold and bootstrap values $\geq 90$ are indicated by dots.

\section{Supplementary Files}

This is a list of supplementary files associated with this preprint. Click to download.

- 2022ScyllametagenomeSupplementME.pdf 